\title{
Elargissement du réseau de nos sociétés fiduciaires partenaires dans le canton de Genève
}

FMH Services présente dès le $1^{\text {er }}$ novembre 2014 un nouveau partenaire de confiance FMH Fiduciaire Services à Genève.

ACTA SA, société fiduciaire agréée ASR en qualité d'expert-réviseur sous le numéro 504662, a pour vocation de proposer à ces clients une large gamme de services allant de la simple tenue comptable aux missions de révision et d'audit en passant par les travaux d'expertise comptable, d'établissement de comptes annuels ou de déclaration fiscale.

Fondée et dirigée par Messieurs Stanley Kumar et Daniel Humbel, respectivement expert comptable et expert-réviseur agréé, ACTA SA est très attachée au sens du service.

Présents sur le terrain, proches de leurs clients et toujours à leur disposition, les collaborateurs d'ACTA SA ont pour objectif d'entretenir une relation personnalisée avec leurs clients qui sont, pour la plupart, dirigeants et chefs d'entreprise.

ACTA SA bénéficie d'une grande expérience dans le domaine médical en réalisant une large palette de prestations, notamment:

- Tenue comptable et fiscale auprès des professionnels de la santé

- Révision au sens de l'Autorité de Surveillance en matière de Révision (ASR) de plusieurs cliniques et centres médicaux

- Etude de financement

- Assistance lors de la création et de la remise de cabinet médical

- Planification retraite et cessation d'activité

Les membres fondateurs d'ACTA SA restent fidèles à leur ligne de conduite originelle: mettre leurs compétences au service des personnes qui les consultent, tout en privilégiant le dialogue.

\section{ACTA SA}

FMH Fiduciaire Services Rue De-Candolle 11 1205 Genève

Tél. +41 (0)22 7005640

Fax +41 (0) 219632166

www.fmhfiduciaire.ch

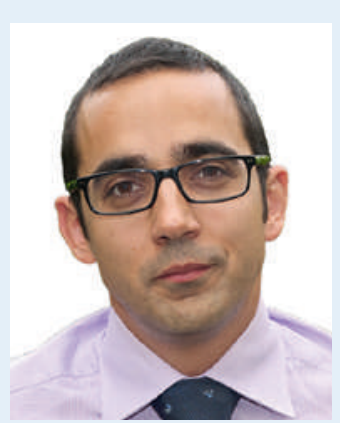

Stanley Kumar, expert comptable et réviseur agréé

stanley.kumar@fmhfiduciaire.ch

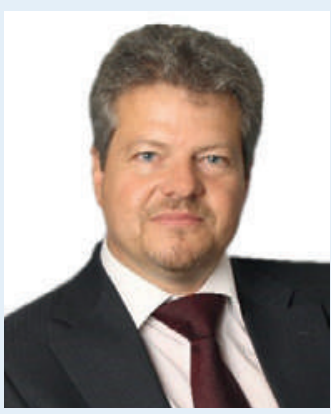

Daniel Humbel, expert comptable diplômé et expert-réviseur agréé daniel.humbel@fmhfiduciaire.ch

\section{Problèmes de TVA?}

«Les prestations médicales ne sont pas soumises à la TVA!» Si cette affirmation est correcte, elle ne l'est cependant pas dans tous les cas. C'est pourquoi il convient d'étudier la question en détail dans le cadre de cabinets doubles ou de groupe ou encore de la propharmacie. En tant que spécialistes, les partenaires de confiance FMH Fiduciaire Services se tiennent à votre entière disposition. N'hésitez pas à faire appel à eux.

FMH Fiduciaire Services

Burghöhe 1, 6208 Oberkirch

Tél. 0419250077 - Fax 0419210586

mail@fmhfiduciaire.ch - www.fmhfiduciaire.ch 